\title{
GRAVITATIONAL REDSHIFTS AND THE MASS-RADIUS RELATION
}

\author{
Gary Wegner \\ Department of Physics \& Astronomy, Dartmouth College
}

\section{INTRODUCTION}

The gravitational redshift is one of Einstein's three original tests of General Relativity and derives from time's slowing near a massive body. For velocities well below c, this is represented with sufficient accuracy by:

$$
\mathrm{V}_{\mathrm{RS}}=\mathrm{GM} / \mathrm{cR}=0.635 \mathrm{M} / \mathrm{M}_{\odot} / \mathrm{R} / \mathrm{R} \odot \mathrm{km} / \mathrm{sec} .
$$

As detailed by Will (1981), Schiff's conjecture argues that the gravitational redshift actually tests the principle of equivalence rather than the gravitational field equations. For low redshifts, solar system tests give highest accuracy. LoPresto \& Pierce (1986) have shown that the redshift at the Sun's limb is good to about $\pm 3 \%$. Rocket experiments produce an accuracy of $\pm 0.02 \%$ (Vessot et al. 1980), while for 40 Eri B the best white dwarf, the observed and predicted VRS agree to only about $\pm 5 \%$ (Wegner 1980).

While using white dwarfs is not the most stringent test of general relativity, one can assume its validity and employ it as a tool to probe the white dwarfs. Here, the mass-radius relation is examined and then used to constrain non-Newtonian gravitational force laws.

\section{PRESSURE SHIFTS IN THE LINES}

The role of pressure shifts is important for interpretating white dwarfs' radial velocities. These appear to be manageable for pure hydrogen atmospheres, but many difficulties remain for the helium dominated atmospheres.

For hydrogen, it has long been known that in high density plasmas, the Balmer lines show assymetries. (Shipman \& Meehan 1976). Recent work of Grabowski et al. (1987), however shows that the velocities measured depend on resolution. The narrow cores of the Balmer lines are relatively unaffected, while in the line wings, there are assymetries that could lead to redshifts of the size found in the early work described in $\$ 2.2$. Consequently, if measurements are made in the line cores at sufficiently high resolution, pressure shifts can be ignored.

For He I, there is much uncertainty. Greenstein \& Trimble (1967), Wegner (1973), and Koester (1987) all point out that each individual line gives its own redshift in the spectra of the DBs. Additional laboratory and theoretical work are needed to clarify this problem before the He I lines of DB stars can be reliably used. A possible project would be to use the $H \alpha$ line in DBA stars to calibrate them. Hammond's recent work for Ca II reported in these proceedings suggest that this problem is tractable for the DZ stars. 


\section{WHITE DWARF GRAVITATIONAL REDSHIFT MEASUREMENTS}

\subsection{Individual Stars with well-known Orbits.}

The earliest attempt to observe any gravitational redshift was for Sirius B by Adams (1925). However, this value is questionable due to problems with scattered light from $A$. Greenstein, Oke, \& Shipman (1971) derived $V_{R S}=89 \pm 16 \mathrm{~km} / \mathrm{sec}$. Indeed, Sirius $B$ has proven difficult in the visual due to the proximity of Sirius $A$ and several investigators including the author have attempted measurements and failed. The mass is well established as $1.053 \pm 0.028$ M० (Gatewood \& Gatewood 1978), but there are still questions about the radius (cf. Kidder, Holberg, \& Wesemael these proceedings).

The white dwarf most amenable to direct comparison of $M, R$, and the redshift is $40 \mathrm{Eri}$ B. Popper's (1954) classical study of the star yielded the often quoted value of $+21_{ \pm} 4 \mathrm{~km} / \mathrm{sec}$. Greenstein \& Trimble (1972) found a redshift of $+23_{ \pm} 5 \mathrm{~km} / \mathrm{sec}$ for this object. More recent work by Wegner $(1979,1980)$ suggests a slightly higher value, viz. $+23.9 \pm 1.3 \mathrm{~km} / \mathrm{sec}$, which may be inconsistent with the astrometric mass of $0.44 \mathrm{M} \odot$.

Other binaries may be usable for determining gravitational redshifts, but present additional problems. Procyon $B$ has never been measeured, while Stein 2051 (Strand \& Kallarkal these proceedings) and G107-70 (Harrington et al. 1981) have orbits, but they are of spectral class DC and require examination at higher signal-to-noise and resolution.

\subsection{Statistical Methods}

The determination of the $K$-term for the white dwarfs yields a mean gravitational redshift through the relation:

$$
R V O b s=K-S \cos \lambda+V p e c
$$

This approach was pioneered by Greenstein \& Trimble (1967) and Trimble \& Greenstein (1972) who obtained $K=+65 \pm 5 \mathrm{~km} / \mathrm{sec}$, which is discrepant with the mean mass near $0.6 \mathrm{M \odot}$ found from using $\log g$ which should yield a mean redshift near $+31 \mathrm{~km} / \mathrm{sec}$ for a carbon composition.

More recent studies suggest a lower $K$. Wegner (1974) observed southern white dwarfs and derived $+43 \pm 14 \mathrm{~km} / \mathrm{sec}$ and even more recently, Greenstein et al. (1977) obtained $+44.6 \pm$ $5.7 \mathrm{~km} / \mathrm{sec}$ from high resolution data of 13 stars.

\subsection{Common Proper Motion Systems}

Common proper motion (CPM) binaries containing a white dwarf are numerous and using them to study redshifts seems most promising. If such a system is really gravitationally bound, the orbital contribution to the observed radial velocity is typically of order \pm 1 $\mathrm{km} / \mathrm{sec}$, below the usual precision of measurement. Often, however, these systems are more distant and have more uncertain distances.

Nevertheless, large numbers of CPM objects exist and the first lists were compiled by Luyten (1969) and used by Eggen \& Greenstein (1965) to study white dwarfs. A few individual redshifts were obtained by Greenstein \& Trimble (1967) and Trimble \& Greenstein (1972). More recently there has been a renewed interest in these systems and the article by Sion \& 
Oswalt in these proceedings contains further references to Oswalt's and other work cataloging CPM systems containing white dwarfs,

The first systematic attempt to utilize the brighter systems was Wegner (1973) who obtained image-tube spectra of the white dwarf members and high resolution photographic spectra of the bright non-degenerate components of 5 systems in the southern hemisphere. These data were used to further obtain evolutionary parameters for the white dwarfs. Sion \& Guinan (1985) have applied this technique to the DO white dwarf HD149499 using IUE Wegner's (1978a) radial velocity for $A$ to derive $V_{R S}= \pm 17+5 \mathrm{~km} / \mathrm{sec}$.

The star CoD-380 10980 is an example of how things can go wrong (Holberg et al. 1985). Here, the distance is not well known despite several parallax measures and the redshift, near $+40 \mathrm{~km} / \mathrm{sec}$ (Wegner 1978b; Koester 1987) disagrees with that predicted from logg.

Nevertheless, recent application of modern digital detectors has yielded greatly improved accuracy for the measurements of redshifts. The values derived in Koester (1987), Wegner \& Reid (1987) and elsewhere give both much improved internal and external accuracy.

\subsection{White Dwarfs in the Hyades and Other Galactic Clusters}

White dwarfs in galactic clusters represent another method of obtaining gravitational redshifts with the added advantage of a known cluster age. However, there can be kinematical complications and most galactic clusters are too distant for high resolution studies of the white dwarf members.

The Hyades is the nearest with a number of white dwarf members at about the 14th magnitude. These stars are discussed elsewhere in these proceedings (Wegner, Reid, \& McMahan 1988).

The next nearest clusters include the Pleiades and Praesape. The one Pleiad with $V=$ 16.5 is correspondingly more difficult, so far has defying recent efforts at determining a redshift. Praesape (Luyten 1962) should be reachable with 5-m class instruments. A number of even fainter white dwarfs have been located in other clusters, notably through the efforts of Koester \& Reimers (1985 and refs. therein), Anthony-Twarog (1982), and Romanishin \& Angel (1980) but these are currently unreachable at sufficiently high resolution and signalto-noise for redshifts.

\section{THE MASS-RADIUS RELATION AND THE MASS DISTRIBUTION}

The theoretical mass-radius relation (Chandrasekahr 1934) has stood the test of time well, despite many subsequent elaborations on the equation of state, but it has never been closely verified empirically as discussed by Weidemann in these proceedings. The richness of details that can be learned is obvious and deserves study.

Except for the few visual binary white dwarfs, the mass-radius relation must be tested by working with combinations of observed quantities, e.g. gravity $g=G M / R^{2}$ or redshift, $V_{R} S$ $=0.635(\mathrm{M} / \mathrm{M} \odot) /(\mathrm{R} / \mathrm{R} \odot) \mathrm{km} / \mathrm{sec}$. Using $g$ suffers from large errors. McMahan (1988) has reduced the uncertainties, but for studying individual stars in the mass-radius relation, 
errors of order \pm 0.05 dex are needed in both $\log g$ and $\log R$. However, as an error of \pm 3 $\mathrm{km} / \mathrm{sec}$ translates to about $\pm 0.03 \mathrm{M} \odot$, it appears possible to make some meaningful conclusions utilizing gravitational redshifts.

Table I presents data derived from the presently best available $V_{R S}$, taken from Wegner \& Reid (1987), Koester (1987)in addition to the stars in Wegner, Reid \& McMahan (1988), and references in $\S 2.1$. Sirius B is plotted using Gatewood \& Gatewood (1978) for the mass. The radii have been taken from sources in Wegner, Reid, \& McMahan (1988), except Sirius B where Thejll \& Shipman (1986) was used. Masses correspond to the redshifts if the stars lie on the carbon Hamada \& Salpeter (1961) mass-radius relation. Weights are assigned as in the Hyades paper.

\section{TABLE I}

Data on White Dwarfs in Binaries with Reliable Redshifts

\begin{tabular}{|c|c|c|c|c|c|c|c|c|c|}
\hline Name & $\mathrm{V}_{\mathrm{RS}}$ & $\log R / R_{C}$ & $\odot \mathrm{M} / \mathrm{M}_{\odot}$ & Weight & Name & & $\mathrm{gR} / \mathrm{R} \odot \mathrm{I}$ & $\mathrm{L} / \mathrm{M}_{\odot}$ & Weight \\
\hline Sirius B & +89.0 & -2.12 & 1.04 & 4 & G154-B 5A/B & +24.5 & -1.91 & 0.51 & 2 \\
\hline 40 Eri $B$ & +23.9 & -1.87 & 0.50 & 19 & $G 200-39 / 40$ & +32.5 & -1.98 & 0.61 & 2 \\
\hline L970-27/30 & +19.9 & -1.88 & 0.44 & 4 & L587-77A & +36.1 & -1.87 & 0.66 & 2 \\
\hline $\mathrm{W} 485 \mathrm{~A} / \mathrm{B}$ & +24.9 & -1.83 & 0.52 & 4 & LDS455 & +51.2 & -2.07 & 0.80 & 2 \\
\hline $\mathrm{W} 672 \mathrm{~A} / \mathrm{B}$ & +21.2 & -1.84 & 0.46 & 4 & $L 481-60$ & +27.9 & -1.84 & 0.56 & 2 \\
\hline G142-B2A/B & +27.4 & -1.94 & 0.55 & 2 & $C D-38: 10980$ & +40.0 & -1.93 & 0.69 & 4 \\
\hline G148-6/7 & +27.0 & -1.91 & 0.54 & 2 & L268-92 & +30.2 & -1.88 & 0.59 & 2 \\
\hline
\end{tabular}

Figure 1 presents a histogram of the masses from Table I and the Hyades in Wegner, McMahan, \& Reid (these proceedings). The masses have been derived from VRS on the assumption that each star lies on the Hamada \& Salpeter (1961) carbon mass-radius relation. Although the number of objects is still small, the field stars reproduce the relation discussed in Prof. Weidemann's lecture. The difference in unwieghted mean mass for the Hyades (0.66 $\pm 0.05 \mathrm{M} \odot)$ and the field objects $\left(0.57 \pm 0.03 \mathrm{M}_{\odot}\right.$ excluding Sirius $\left.\mathrm{B}\right)$ seems to be significant and could show real differences in the parent star masses.

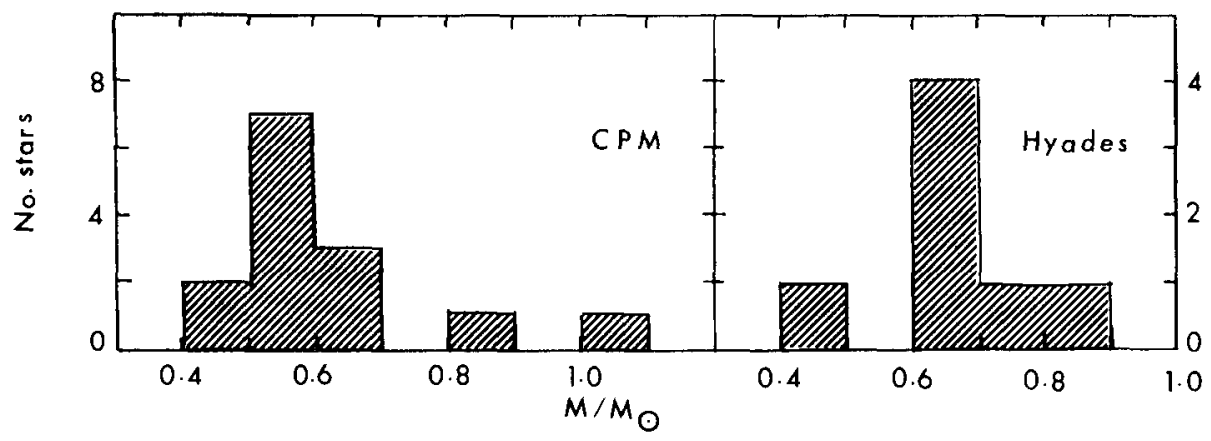

FIG. 1. The distribution of masses for white dwarfs from the gravitational redshifts published in Koester (1987), Wegner \& Reid (1987), and Wegner, Reid, \& McMahan (1988), derived on the assumption that all stars lie on the Hamada-Salpeter carbon mass-radius relation. 
The center panel of Figure 2 shows all stars in Figure 1 compared with the Hamada \& Salpeter (1961) C mass-radius relation. The data points are fit reasonably well and this is clearly better than that given by $n=1.5$ polytropes. The scatter is larger than expected from errors in $V_{R S}$ and this could reflect imprecision in $\log R$ arising through the distance determinations.

Future work must include an assessment of the role of spectroscopic binary white dwarfs like L870-2 (Cf. Bragaglia et al. and Saffer \& Liebert, these proceedings) on enhancing the scatter in the mass determinations.

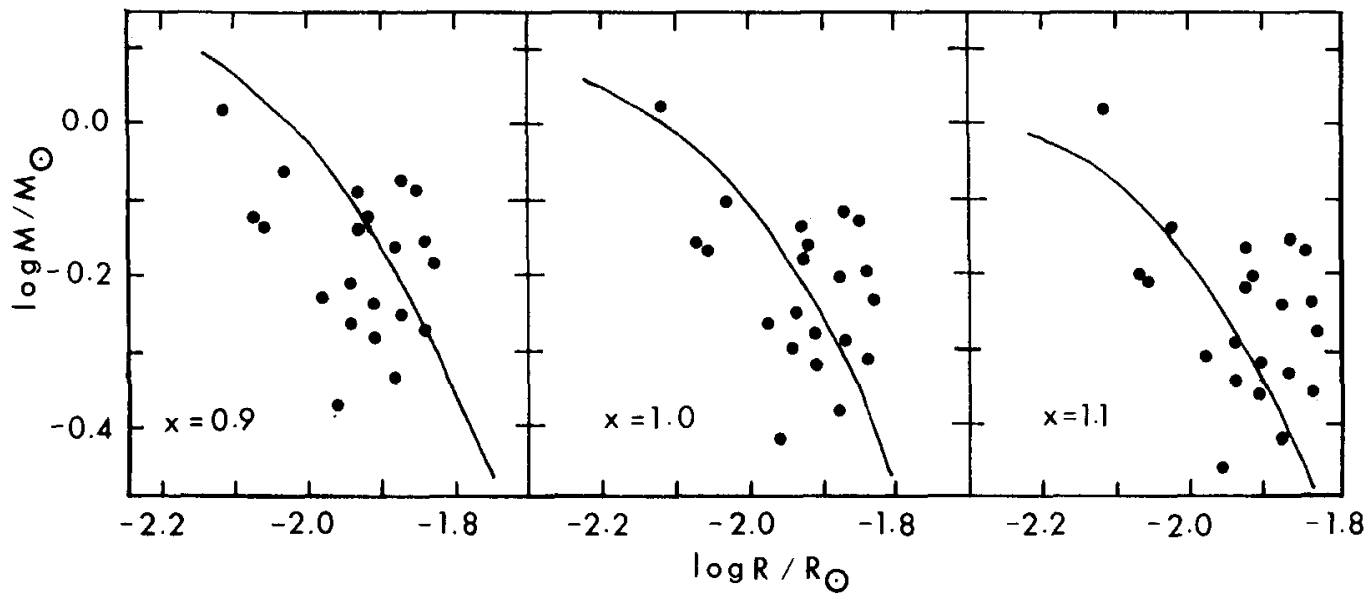

FIG. 2. Positions of the white dwarfs with the best observed gravitational redshifts compared with the Hamada-Salpeter pure carbon mass-radius relation, scaled for different values of the gravitational constant as given by the parameter $x=G_{o} / G_{\infty}$.

\section{CONSTRAINTS ON THE DISTANCE DEPENDANCE OF GRAVITY}

Although white dwarfs cannot compete with other measurements of the gravitational redshift as tests of general relativity, they can still be used to study certain aspects of the gravitational force law. Extended supergravity theories predict the addition of a Yukawa term to the usual Newtonian gravitational potential, so that

$$
U(r)=-G_{\infty} M / r\left(1+\alpha e^{-r / \lambda}\right)
$$

with the resulting modified gravitational attraction, $F=G(r) M / R$, with $G(r)=G_{\infty}\{1+\alpha(1+$ $\left.R / \lambda) \mathrm{e}^{-\mathrm{r} / \lambda}\right\}$.

For values of $\lambda$ between a few $\mathrm{m}$ to $\mathrm{km}$, empirically determining $\alpha$ has proven difficult and the results are presently controversial. Long (1976) described experimental measures of $\alpha$ and more recently, the work of Echardt et al. (1988), Thielberger (1987) and Stubbs et al. (1987) using terrestrial experiments have found differing results at about the few per cent level.

For white dwarfs the equation of state is well known and stellar evolution establishes that they should mostly have CO cores. Sugimoto (1972) and Blinnikov (1978) made use of the fact that for the Chandrasekhar (1934) mass-radius relation, the mass and radius have the 
following scaling with gravity :

$$
M_{\infty}=M_{0} x^{-1.5} \text { and } R_{\infty}=R_{0} x^{-0.5} \text {, where } x=\left(G_{\infty} / G_{0}\right) .
$$

Hut (1981) argued that the corresponding variation in the Hamada \& Salpeter (1961) massradius relation also scales with $G$ to about $0.2 \%$ and found from Sirius $B$ that $x=0.98 \pm 0.08$. As well, 40 Eri B gives a similar result (Wegner 1980).

The limits on $\mathrm{x}$ can be diminished using the radii and masses of white dwarfs from their gravitational redshifts. Both $V_{R S}$ and $\log R / R_{\odot}$ are observed quantities and do not scale with $x$. The derived mass will be:

$$
\mathrm{M} / \mathrm{M} \odot=\mathrm{V}_{\mathrm{RS}}(\mathrm{R} / \mathrm{R} \odot) / 0.635 / \mathrm{x} .
$$

Consequently, for each value of $\mathrm{x}$, there is a mass-radius relation and a corresponding point for each of the white dwarfs.

Using the data in Figure 2 from $\S$ IV and scaling the Hamada \& Salpeter (1961) carbon mass-radius relation with $x$ as above, a least squares best fit yields $x=0.994 \pm 0.012$ (s.d). For Sirius B, the position was fixed using the Gatewood \& Gatewood (1978) mass and Thejll \& Shipman's (1986) radius. The possible complications of assuming the zero-temperature $C$ mass-radius relation must be emphasized at this point. Nevertheless, this derived value of $\mathrm{x}$ is compatable with terrestrial experiments that find $\mathrm{x}$ differing little from unity and has an accuracy comparable to those investigations.

\section{CONCLUSIONS}

While the results described in this article are preliminary due to the small numbers of stars available, the use of gravitational redshifts as a probe of the properties of white dwarfs seems most promising. Recent advances in digital detectors has made this possible and detailed data on mass distributions can be expected as measurements for more systems become available. White dwarfs can also probe the gravitational force and do not seem to support large deviations from the Newtonian law.

This work was partially supported by the National Science Foundation through Grant AST85-15219.

\section{REFERENCES}

Adams, W. S. 1925 Proc. Nat. Acad. Sci., 11, 382.

Anthony-Twarog, B. J. 1982, Ap. J., 255, 245.

Blinnikov, S. I. 1978, Astrophys. Space Sci., 59, 13.

Chandrasekhar, S. 1934, M. N. R. A. S., 95, 207.

Echardt, D., Jekeli, C., Lazarewicz, A., Romaides, A. \& Sands, R. 1987, Phys. Rev. Lett., $56,3$.

Eggen, O. J. \& Greenstein, J. L. 1965, Ap. J., 141, 83.

Gatewood, G. D. \& Gatewood, C. V. 1978, Ap. J., 225, 191.

Grabowski, B., Madej, J., \& Halenka, J. 1987, Ap. J., 313, 750.

Greenstein, J. L., Boksenberg, A. L., Carswell, R., \& Shortridge, K. 1977, Ap. J., 212, 186.

Greenstein, J. L., Oke, J. B., \& Shipman, H. L. 1971, Ap. J., 169, 563.

Greenstein, J. L. \& Trimble, V. L. 1967, Ap. J., 149, 283. 
Hamada, T. \& Salpeter, E. E. 1961, Ap. J., 134, 683.

Harrington, R. S., Christy, J. W., \& Strand, K. Aa. 1981, A. J., 86, 909.

Holberg, J. B., Wesemael, F., Wegner, G., \& Bruhweiler, F. C. 1985, Ap. J., 293, 294.

Hut, P. 1981, Phys. Lett. B, 99B, 174.

Koester, D. 1987, Ap. J., 322, 852.

Koester, D. \& Reimers, D. 1985, Astr. Ap., 153, 260.

Long, D. R. 1976, Nature, 260, 417.

LoPresto, J. C. \& Pierce, K. 1986, Bull. Am. Astr. Soc., 18, 989.

Luyten, W. J. 1962, A Search for Faint Blue Stars XXXI. One Thousand Blue Stars in the Region of Praesepe, (Univ. of Minnesota: Minneapolis).

Luyten, W. J. 1969, Proper Motion Survey with the Forty-eight Inch Schmidt Telescope, XVII, Binaries with White Dwarf Components, (Univ. Minnesota: Minneapolis).

McMahan, R. K. 1988, Ap. J., in press.

Popper, D. M. 1954, Ap. J., 120, 316.

Romanishin, W. \& Angel, J. R. P. 1980, Ap. J., 235, 992.

Sion, E. M. \& Guinan, E. F. 1985, Ap. J. (Letters), 265, L87.

Stubbs, C., Adelberger, E., Raab, F., Gundlach, J., Heckel, B., McMurray, K., Swanson, H., \& Watanabe, R. 1987, Phys. Rev. Lett., 58, 1070.

Sugimoto, D. 1972, Prog. Theor. Phys., 48, 699.

Thieberger. P. 1987, Phys. Rev. Lett., 58, 1066.

Thejll, P. \& Shipman, H. L. 1986, P. A. S. P., 98, 922.

Trimble, V. L. \& Greenstein, J. L. 1972, Ap. J., 177, 441.

Vessot, R. F. C. et al. 1980, Phys Rev. Lett., 45, 2081.

Wegner, G. 1973, M. N. R. A. S., 165, 271.

Wegner, G. 1974, M. N. R. A. S., 166, 271.

Wegner, G. 1978a, M. N. R. A. S., 182, 111.

Wegner, G. 1978 b, M. N. R. A. S., 187, 17.

Wegner, G., 1979, A. J., 84, 650.

Wegner, G., 1980, A. J., 85, 1255.

Wegner, G. \& Reid, I. N. 1987, in Proc. IAU Colloq. 95. The Second Conference on Faint Blue Stars, ed. A. G. D. Philip, D. S. Hayes, \& J. W. Liebert, (L. Davis Press: Schenectady), p. 649.

Wegner, G., Reid, I. N., \& McMahan, R. K. 1988, These proceedings.

Will, C. M. 1981, Theory and Experimentation in Gravitational Physics, (Cambridge University Press: Cambridge), p. $38 \mathrm{ff}$. 